\title{
Motivating and assisting physical exercise in independently living older adults: A pilot study
}

\author{
Patrícia Silveira ${ }^{a, *, 1}$, Eva van het Reve $e^{b, 2}$, Florian Daniel ${ }^{a, 1}$, Fabio Casati ${ }^{a, 1}$, \\ Eling D. de Bruin ${ }^{b, 2}$ \\ a University of Trento, Via Sommarive, 14 I-38123 Povo, Trento, Italy \\ ${ }^{\mathrm{b}}$ Institute of Human Movement Sciences and Sport, Department Health Sciences and Technology, ETH Zürich, Wolfgang-Pauli-Str. 27, \\ 8093 Zürich, Switzerland
}

\section{A R T I C L E I N F O}

\section{Article history:}

Received 11 June 2012

Received in revised form

26 November 2012

Accepted 27 November 2012

\section{Keywords:}

Physical exercises

Motivation

Elderly

Tablet

Mobility

Healthcare

\begin{abstract}
A B S T R A C T
Background: With age reaction time, coordination and cognition tend to deteriorate, which may lead to gait impairments, falls and injuries. To reduce this problem in elderly and to improve health, well-being and independence, regular balance and strength exercises are recommended. However, elderly face strong barriers to exercise.

Objective: We developed Active Lifestyle, an IT-based system for active and healthy aging aiming at improving elderly's balance and strength. Active Lifestyle is a proactive training application, running on a tablet, which assists, monitors and motivates elderly to follow personalized training plans autonomously at home, while integrating them socially. The objective is to run a pilot study to investigate: (i) the feasibility of assisting the autonomous, physical training of independently living elderly with the Active Lifestyle system, (ii) the adherence of the participants to the training plans, and (iii) the effectiveness of the motivation instruments built into the system.

Methods: After three introductory meetings, 13 elderly adults followed personalized twoweeks strength and balance training plans using the Active Lifestyle app autonomously at home. Questionnaires were used to assess the technological familiarity of the participants, the feasibility aspects of the physical intervention, and the effectiveness of the motivation instruments. Adherence to the exercise plan was evaluated using the performance data collected by the app during the study.

Results: A total of 13 participants were enrolled, of whom 11 (85\%) completed the study (mean age $77 \pm 7$ years); predominantly females (55\%), vocational educated (64\%), and their past profession requiring moderate physical activity (64\%). The Active Lifestyle app facilitated autonomous physical training at home (median $=7$ on a 7-point Likert scale), and participants expressed a high intention to use the app also after the end of the study (median $=7$ ). Adherence with the training plans was $73 \%$ ( $89 \%$ on the balance exercises and $60 \%$ on the strength exercises). The outcome from our questionnaires showed that without the app the
\end{abstract}

Abbreviations: App, application; ATM, automatic teller machine; CD, compact disk; ETH, eidgenössische technische hochschule; GPS, global position system; IT, information technology.

* Corresponding author. Tel.: +390461 282093.

E-mail addresses: silveira@disi.unitn.it (P. Silveira), eva.vanhetreve@hest.ethz.ch (E.v.h. Reve), daniel@disi.unitn.it (F. Daniel), casati@disi.unitn.it (F. Casati), eling.debruin@hest.ethz.ch (E.D. de Bruin).

1 Tel.: +390461282093.

2 Tel.: +41446324018.

1386-5056/\$ - see front matter @ 2012 Elsevier Ireland Ltd. All rights reserved.

http://dx.doi.org/10.1016/j.ijmedinf.2012.11.015 
participants did not feel motivated to perform exercises; with the support of the app they felt more motivated (median=6). Participants were especially motivated by being part of a virtual exercise group and by the capability to automatically monitor their performance (median $=6$ for both).

Conclusions: This study shows that the Active Lifestyle app prototype has valuable potential to support physical exercise practice at home and it is worthwhile to further develop it into a more mature system. Furthermore, the results add to the knowledge base into mobile-based applications for elderly, in that it shows that elderly users can learn to work with mobile-based systems. The Active Lifestyle app proved viable to support and motivate independently living elderly to autonomously perform balance and strength exercises.

(c) 2012 Elsevier Ireland Ltd. All rights reserved.

1.

\section{Introduction}

Health status is an important indicator of quality of life among older persons [1,2]. Functional performance, chronic conditions, and diseases, which directly influence fitness, are related to the perceived health among middle-aged and older adults [2-4]. Chronic diseases are, furthermore, leading causes of death and disability in both developed and developing countries [5,6]. Inactivity is at the origin of several chronic diseases [7]. Regular physical activity or exercise substantially prevents the development and progression of most chronic degenerative diseases [8-10], is of benefit to frail and older persons, and is the only therapy found to consistently improve sarcopenia, physical function, cognitive performance and mood in older adults [11]. For older people, a sedentary lifestyle also increases the risk of falls, whereas physically active older people have a reduced risk of falls with injuries [12-14]. In summary, it is evident that to increase older adults' quality of life and fitness, we need to encourage the elderly to become more physically active $[15,16]$.

There are a variety of barriers that make it hard for elderly to maintain or increase their physical activity level. Neighborhoods and communities may be poorly designed or perceived as being unsafe, thus preventing elderly from leaving home $[17,18]$. Older adults may have trouble getting to specialized facilities (e.g., community center for the aged) and physical training programs offered in such institutions $[17,18]$. General health care professionals (e.g., nurses, family physicians) may lack the time or expertise to address problems of physical inactivity among their older patients, and often lack information about quality programs, training materials, and how to make referrals to community resources [19]. Furthermore, elderly often express the desire for training support at home [20].

Specialized healthcare professionals (i.e., sport medicine, gerontologists, physiotherapists and human movement scientists) strongly recommend physical training programs that focus on muscle strength and balance [21] to promote health, well-being, and functional independence of the elderly. Structured exercise training has a positive impact on older adults and may be used for the management of frailty [22-24]. Home environmental interventions based on different forms of assistive technology devices have, in this context, the potential to overcome some of the barriers to start training and maintain physical independence for independent living elderly [25]. However, the effectiveness of such an approach has not yet been demonstrated to a large extent. Modern exercise equipment may not always be suitable for elderly individuals, who might be concerned about the intensity of training sessions and may rather express to have a preference for more traditional therapy approaches [26]. New treatments usually have to go through a series of phases to test whether they are feasible, safe and effective [27]. It seems, therefore, necessary to perform a pilot study to assess the feasibility of applying assistive technology devices in an elderly population with the aim to encourage performance of targeted physical exercise. Findings of such a study can inform a larger scale main Phase III study [27].

The objective of this study is to run a Phase II pilot study according the model for complex interventions advocated by the British Medical Research Council [28] with an iPad-based app (short for application) called Active Lifestyle, a software for the autonomous physical training of strength and balance for independently living elderly. We aim to investigate (i) the feasibility of assisting the autonomous, physical training of independently living elderly with the Active Lifestyle system, (ii) the adherence of the participants to the Active Lifestyle training plans, and (iii) the effectiveness of the system's motivation instruments.

\section{Methods}

\subsection{The Active Lifestyle app}

Active Lifestyle is a pro-active software for active and healthy aging, assisting and monitoring elderly during autonomous home-based physical workouts [29,30]. The interface is designed to be simple and avoid frustration generated by the inability to use technology, and uses persuasive strategies to motivate elderly users to keep a routine of physical exercises.

The app supports strength and balance training plans. The strength training should be done twice a week; it starts with 6 warm-up exercises, and is followed by 9 strength and 3 stretching exercises. There is a minimum number of sets (1-3) and repetitions (15-30) for each exercise. Some exercises also require the use of weights $(2-6 \mathrm{~kg})$. The balance training should be done five days per week. Each session is composed of 3 exercises, in which the elderly repeatedly (1-3 times) holds a certain position for several seconds (15-30s). The training program follows best practices recommendations [31] and generally accepted training principles (e.g., is progressive in nature) [32]. An example of exercises is illustrated in Fig. 1 and the training procedure is presented in Fig. 2 and in a video [33]. 


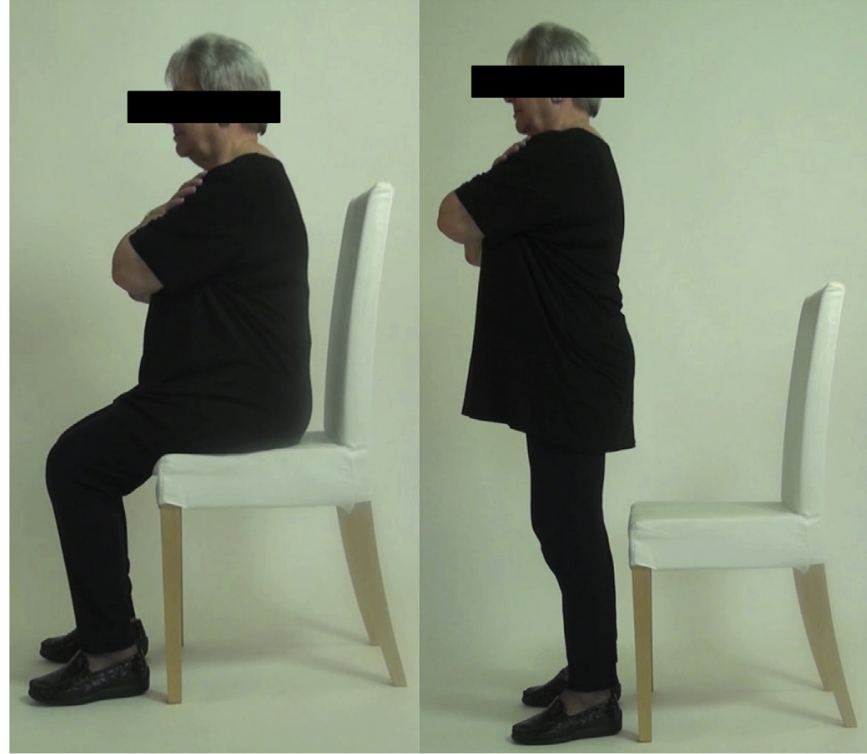

(a)

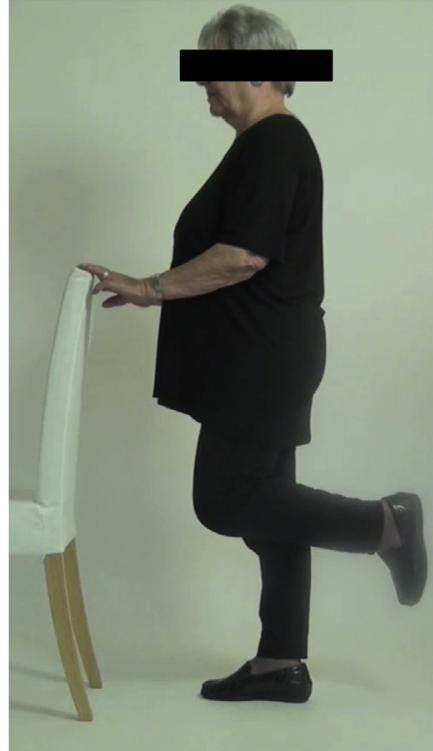

(c)

Fig. 1 - (a) and (b) illustrate the Chair stand exercise (strength); (c) shows the One leg stand exercise (balance).

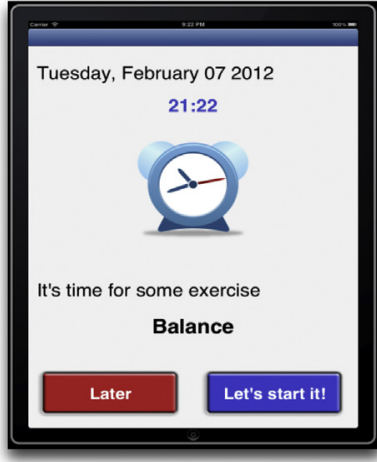

a) An alarm sounds three times per day to warn the user about the planned workout sessions.

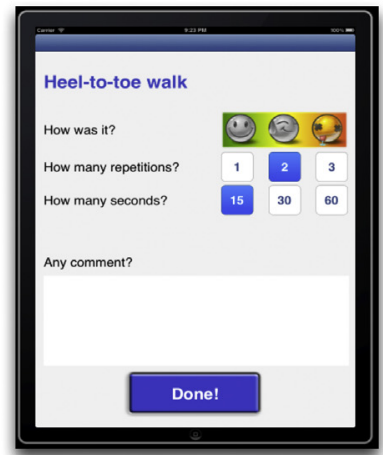

d) After each exercise, the user provides feedback on his/her performance.

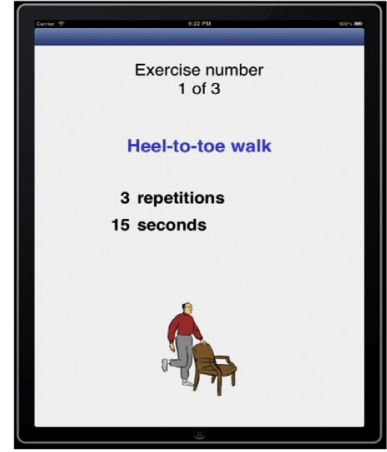

b) Spoken and written instructions introduce the exercises.

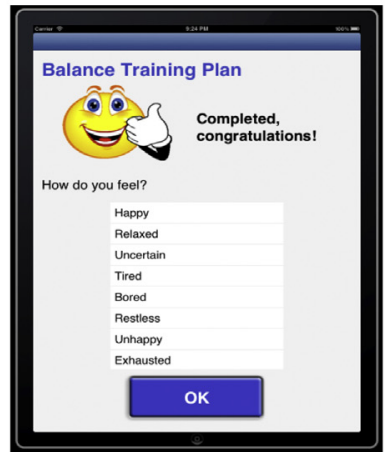

e) At the end of a workout session, the user enters his/ her mood.

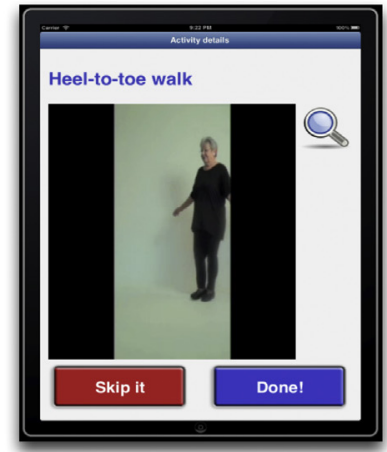

c) Video and step-by-step instructions guide the user through each exercise.

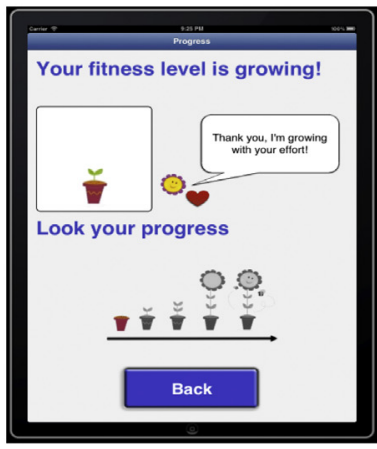

f) Finally, the progress of the user is showed through a flower metaphor.

Fig. 2 - Main screenshots of the training plan procedure. 
To motivate the elderly, the app supports individual and social motivation instruments [34].

Individual motivation strategies aim at convincing someone to do something by making it enjoyable for this person, independently of any social pressure. The app specifically supports:

- Conditioning through positive and negative reinforcement: that is, immediately offering a reward/praise after an expected behavior to encourage the behavior and to increase the probability that it happens again, or reprimanding whenever undesired behavior happened aiming to decrease the probability of a reoccurrence of the behavior. We use metaphors for positive and negative reinforcement, i.e., a flower that grows whenever a session is completed (i.e., positive reinforcement) and that has a mood status that varies according to the person's daily compliance to the plan (i.e., positive and negative reinforcement, if the person performs the exercise the flower is happy, otherwise it is sad) (see Fig. 3a). The flower does not die or become ugly to avoid possible negative reactions on the users;

- Goal-setting: establishing specific, measurable, achievable, and time-targeted goals. We communicate the goal by anticipating the best achievable growth of the flower metaphor (Fig. 3b);

- Self-monitoring: allowing people to monitor themselves and to modify their attitudes and behaviors. Coloring the respective flower growth stages reflects progress toward the goal (Fig. 3b).

- Social motivation strategies are built on social psychology. An individual's social network (other people participating in the same training) is the source of motivation, e.g., Active Lifestyle uses:

- Comparison: allowing a person to compare similarities and differences between two or more parties, people tend to keep equality in their relationships. Whenever a person completes a workout session, an automatic message is posted on a Bulletin Board informing the training community (i.e., other users following the same training plans). The message also shows the status of the individual's flower metaphor.

- External monitoring: allowing one party to monitor the behavior of another party to modify behavior in a specific way. In Active Lifestyle, healthcare and IT experts have access to data on performance and compliance toward the plan. The elderly have access to their own flowers and to that of their training partners, so that they can monitor their progress toward the plan too.

\section{Sample}

Participants were 70 years old or older; able to walk independently with or without walking aids; able to follow instructions spoken in German, English, or Italian; and with no severe illness, cognitive impairment, progressive neurological diseases, stroke, severe cardiac failure, or high blood pressure.

\subsection{Setting}

Participants were recruited by convenience sampling (i.e., any subjects willing to participate and matching the selection criteria were accepted) from the "Informationsstelle fuer Altersfragen" in Wollerau, Switzerland. This institution, dedicated to deliver services and information related to aging to the elderly population, issued 220 invitation letters, together with an information sheet outlining the research, inviting independently living elderly of the region to participate. Ethical approval for the study was obtained from the ETH Ethics Committee (EK 2011-N-64).

\subsection{Interventions}

During an initial meeting, all potential participants received information about the Active Lifestyle app and the study. Interested people provided their personal contact data.

During a second meeting, participants were taught how to use the iPad and the app. To ease learning, user guides with written content, illustrations about the app and the iPad were provided.

During a third meeting, participants were taught how to perform the balance and strength exercises supported by the app. The same day, they also signed the consent form and answered health and technology familiarity questions. At the end of this meeting, participants received one resistance band, one Pilates' ball, and one pair of ankle weights $(2 \mathrm{~kg})$. iPads with $3 \mathrm{G}$ SIM-card were lent to the participants. The installed and delivered version of the app contained a predefined training plan community (people participating in the study).

After these three meetings, the participants started a twoweeks home-based balance and strength training. To settle possible remaining obscurities, an additional meeting was scheduled on the second day of the training period. Contact information of our team members was provided to all participants in case of further obscurities or problems.

At the end of the two-weeks training period, a final meeting was held to conclude the study and collect the equipment previously lent. At this time, the participants answered questions regarding the perceived usefulness, usability, visual attractiveness, and the effectiveness of the motivation instruments of the app.

\section{Outcome measures}

The criteria for success, an important part of a pilot study [27], were based on the feasibility of the steps that need to take place as part of a main study and focused on recruitment, attrition and adherence to the exercise. Values for these parameters were compared with median rates in falls prevention interventions in community settings for clinical trials [35], where recruitment of $70 \%$ of the residents that are eligible for the training session, a $10 \%$ attrition rate, and $50 \%$ adherence to the individually targeted exercises were deemed acceptable.

For recruitment, we measured the inclusion rate - i.e. the proportion of participants invited to participate who enrolled into the study - and distinguished between those who refused, 


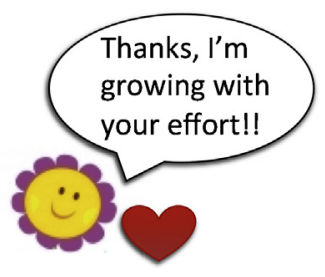

a)

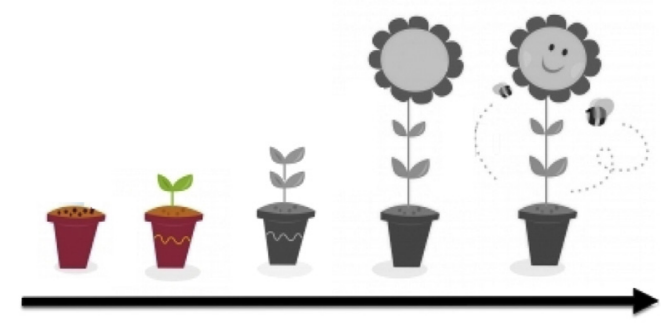

b)

Fig. 3 - Individual motivation instruments based on a flower metaphor.

did not respond or who were willing but excluded (volunteered but did not meet the study inclusion criteria).

For attrition, we measured the number of participants lost at final follow-up.

For adherence to the intervention we recorded engagement with the intervention, e.g., compliance with all four strength and ten balance training sessions. Adherence was computed by Active Lifestyle during the intervention and stored in a central database.

Effectiveness of the motivation instruments built into the system was determined based on the participants' feedback, collected with a 7-Point Likert Scale questionnaire at the end of the intervention. The complete questionnaire, named Feasibility Questionnaire, is available as supplementary material.

Supplementary data associated with this article can be found, in the online version, at http://dx.doi.org/10.1016/ j.ijmedinf.2012.11.015.

\subsection{Statistical analyses}

Descriptive statistics (Mann-Whitney $U$ tests) were used to analyze the questionnaires.

\section{Results}

\subsection{Participant demographics}

Detailed information about the participant demographics is summarized in Table 1. The Health and Technology Familiarity questionnaires used to collect the participants' demographics are available as supplementary material.

Supplementary data associated with this article can be found, in the online version, at http://dx.doi.org/10.1016/ j.ijmedinf.2012.11.015.

Because the rationale for performing this pilot was Process, which deals with the assessment of the feasibility of the steps that need to take place as part of a future main study, we consider feasibility, recruitment, retention and adherence rates as primary findings [27].

\subsection{Feasibility of the Active Lifestyle intervention}

220 information letters were sent. The first information session was held and visited by fourteen residents; all eligible and invited to participate. Thirteen residents consented to join the study resulting in $7 \%$ recruitment rate for the total sample frame. The inclusion rate - fourteen invited to participate; thirteen enrolled - was $93 \%$.

Eleven elderly conducted the two-weeks training resulting in a $16 \%$ attrition rate. Two individuals were lost; one was disappointed about not receiving the iPad after the second meeting and the other because of Wi-Fi connection problems. For adherence to the intervention we had $73 \%$ compliance with all 14 trainings (89\% for balance and $60 \%$ for the strength exercises). There were no reports on adverse events during the training.

Table 1 - Characteristics of the participants.

\begin{tabular}{ll} 
& $\begin{array}{l}\text { Intervention } \\
(n=11)\end{array}$ \\
\hline Female gender, $n$ (\%) & $6 / 11(54.5)$ \\
Mean/median age [range] (years) & $77.2 / 76[70-85]$ \\
Vocational education, $n$ (\%) & $7 / 11(63.6)$ \\
Moderate physical activity in past profession, & $7 / 11(63.6)$ \\
$n$ (\%) & \\
Health questions, $n$ (\%) & $7 / 11(63.6)$ \\
Estimated good health & $5 / 11(45.5)$ \\
Estimated middle balance & $5 / 11(45.5)$ \\
Feeling daily pain & $0 / 11(0)$ \\
Fell in the last six months & $11 / 11(100)$ \\
Leisure time walking at least twice a week & $7 / 11(63.6)$ \\
Practiced some sport in the past & $9 / 11(81.1)$ \\
Never practiced strength exercises & $7 / 11(63.6)$ \\
Wanted to improve fitness & \\
Technology familiarity, $n$ (\%) & $9 / 11(81.8)$ \\
Frequently use ATMs & $5 / 11(45.4)$ \\
Don't use books on tape or CD & $6 / 11(54.5)$ \\
Sometimes use cellphones & $5 / 11(45.5)$ \\
Don't use digital photography & $5 / 11(45.5)$ \\
Don't use electronic book readers & $5 / 11(45.5)$ \\
Don't use GPS & $6 / 11(54.5)$ \\
Don't use automatic kiosks & $9 / 11(81.1)$ \\
Use a computer & $4 / 11(36.3)$ \\
Between 1 and 5 h per week & $9 / 11(81.1)$ \\
Use the Internet & $5 / 11(45.5)$ \\
Between 1 and $5 \mathrm{~h}$ per week & \\
\hline a A fall was defined as unintentionally coming to & the ground or \\
some lower level, excluding the consequence of sustaining a vio- \\
lent blow, loss of consciousness, or sudden onset of paralysis, such \\
as during a stroke or epileptic seizure [36]. \\
\end{tabular}




\subsection{Effectiveness of the Active Lifestyle intervention}

Detailed information about the effectiveness of the Active Lifestyle app and the motivation instruments, as well as the usability aspects are summarized in Table 2.

All participants affirmed that Active Lifestyle facilitates autonomous performance of balance and strength exercises. This is confirmed by a high intention to use the app again or to recommend it to friends or family members $(100 \%$, range $6-7)$. Some participants verbally expressed their disappointment with the end of the training and the impossibility to continue training with it (the app is not yet commercially available).

Participants expressed a high perceived usefulness of the videos. According to some feedbacks, the videos are "absolutely great" and "very helpful." Others stated "I adjusted my posture based on them" or "the exercises were easy with the videos". One explanation for such high approval can be derived from the fact that most of the participants had never performed strength exercises before (82\%).

The Weekly Exercises Calendar - a menu option that allows the user to check the planned workout sessions weekly - was considered useful by all users. The sound alarm was useful to few users only (46\%).

\subsection{Effectiveness of the motivation instruments}

Most of the participants did not feel motivated performing physical exercises in general, however, they felt motivated with the Active Lifestyle app (64\%) because it was fun to follow the exercises with the app (91\%). Their mood after doing the exercises was relaxed (41\%), happy (25\%), tired (19\%), uncertain (9\%), exhausted (3\%), unhappy (1\%), bored (1\%), and restless $(1 \%)$. Values were retrieved from the database based on the participants' feedback after each workout session (Fig. 2e).

The individual motivation instruments were effective. Most participants felt motivated when they saw the flower growing or could monitor their progress toward the plan (both 91\%). At study end we noticed that the participants appreciated the flower metaphor. When filling the questionnaires, most of them made spontaneous comments saying that it was "lovely", "tender and cheerful", or "a sunflower is funny, it makes you happy". However, one woman suggested a racing car instead of the flower. According to her, the car is a perfect metaphor, since it also needs to get warm to work properly. The same high motivation was not achieved with the mood status of the flower (64\%).

The social motivation instruments also achieved good results. The majority of the participants felt motivated by being part of a training plan group (91\%). "I am happy to see others doing the same exercises; I am not a single athlete". Monitoring and comparing the flower were similarly effective (64\%). One woman said "I need someone to push me!" Two participants checked their friends' flowers on the Bulletin Board daily and whether they did the exercises. However, another woman said "Of course not, I am not nosy!" but she also told us "my husband and I always looked at the Bulletin Board to see if there was something interesting to read". The same participants also reported "... after two days I could reach the same level as my husband, it made me happy". From such results, we understood that our participants felt motivated by being part of a social group and by knowing that other people are doing the same exercises and are facing similar challenges (e.g., pain, difficulty wearing the ankle weights). It was, however, possible to notice their interest in knowing how others were doing and comparing other performances with their own. This might be indicative of the human tendency to keep equality (Herzberg's equity theory [37]).

\section{Usability}

All participants were able to use the Active Lifestyle app and agreed that it is easy to use. The scrolling and reading activities were performed by most participants. For instance, navigate through the messages posted on the Bulletin Board using the scroll (91\%); read the posts from the Bulletin Board (100\%); and receive messages on the inBox (73\%, some participants never received a message, so they were not able to express their opinion about this feature). Although the writing activities did not show the same high usage, not all were able to write private messages on the Bulletin Board (64\%) and public messages on the inBox (46\%). Both messages are written following the same approach and appearance. The only difference is that the public messages can be read by all participants, as opposed to the private messages that are only available for selected persons.

A few participants in the beginning felt anxiety at the prospect of using the app (19\%) (e.g., they felt afraid of not being able to use the app correctly or of having problems in using the tablet). With further questions we discovered that one woman had problems to use the app the first day only; after the extra meeting she, however, learned the use and was comfortable with it. Another woman performed the exercises early in the morning when she usually had cold fingers. Due to that, the tablet was not effectively reacting to her touches.

\section{Discussion}

The aim of this study was to investigate (i) the feasibility of the Active Lifestyle system for physical interventions, (ii) the adherence of the participants to the pre-defined training plans, and (iii) the effectiveness of the motivation instruments built into the system.

We demonstrated the feasibility of acquiring acceptable attrition and adherence rates for independently living elderly to the experimental training. Our target of about $70 \%$ recruitment rate (as observed by [35] for similar studies) for the total sample frame, however, by far not met. Those individuals that responded and visited an information session, however, showed a large inclusion and adherence rate. These findings indicate the importance of recruitment strategies and information sessions to create awareness of the need for strength and balance training and the necessary trust for people to also enroll in a study. The differing adherence for the strength and balance training components should be addressed in a revised version of our app. It might be that the balance part required less effort and was easier to perform in contrast to the strength exercises. Many participants made remarks about the load of the strength exercises, e.g., some participants had difficulties 
Table 2 - Outcome data expressed by the participants on a 7-Point Likert Scale (range, 1-7; 1 =completely disagree $7=$ completely agree) at the end of the intervention period $(n=11)$.

\begin{tabular}{|c|c|c|}
\hline Statement & Median (range) & $\begin{array}{l}\text { Percentage } \\
\text { agreed }\end{array}$ \\
\hline $\begin{array}{l}\text { The Active Lifestyle app facilitates the performance of strength and balance exercises autonomously } \\
\text { at home. }\end{array}$ & $7(6-7)$ & 100 \\
\hline \multicolumn{3}{|l|}{ Use intention } \\
\hline I would use the app again & $7(6-7)$ & 100 \\
\hline I would recommend the app to my friends and family. & $6(6-7)$ & 100 \\
\hline \multicolumn{3}{|l|}{ Perceived usefulness } \\
\hline The videos assisted to properly perform the exercises. & $7(6-7)$ & 100 \\
\hline The sound alarm helped to remind me about the planned workout sessions & $4(3-7)$ & 45.4 \\
\hline $\begin{array}{l}\text { The calendar was useful to make me aware about which kind of workout session I need to perform } \\
\text { every day. }\end{array}$ & $7(6-7)$ & 100 \\
\hline \multicolumn{3}{|l|}{ Motivation } \\
\hline I usually don't feel motivated to perform physical exercises, the app helped me & $6(3-7)$ & 63.6 \\
\hline It was funny to me to carry out the strength and balance exercises & $6(3-7)$ & 90.9 \\
\hline I like the pictures of the flower & $7(6-7)$ & 100 \\
\hline I would prefer another picture instead of a flower & $5(2-7)$ & 54.5 \\
\hline \multicolumn{3}{|l|}{ Individual motivation instruments } \\
\hline I felt motivated when I saw the plant growing due to my performance & $6(4-7)$ & 90.9 \\
\hline I felt motivated when I saw my progress on the bar. & $6(4-7)$ & 90.9 \\
\hline I felt motivated when I saw the emotional status of the flower & $6(4-7)$ & 63.6 \\
\hline \multicolumn{3}{|l|}{ Social motivation instruments } \\
\hline It motivated me to be part of a training group and to know that other people did the same exercises & $6(4-7)$ & 90.9 \\
\hline I usually compared my flower with others on the Bulletin Board & $5(2-7)$ & 63.6 \\
\hline I felt motivated to perform the plan because I knew that I was being monitored & $6(2-7)$ & 63.6 \\
\hline \multicolumn{3}{|l|}{ Usability } \\
\hline The operation of the application was easy & $6(5-7)$ & 100 \\
\hline I was able to use the app & $7(6-7)$ & 100 \\
\hline I was able to write messages on the Bulletin Board. & $4(2-7)$ & 45.4 \\
\hline I was able to read messages from the Bulletin Board. & $6(6-7)$ & 100 \\
\hline I was able to send messages to a person (inBox). & $6(4-7)$ & 63.3 \\
\hline I was able to receive messages from a person (inBox) & $6(4-7)$ & 72.7 \\
\hline I was able to navigate through the messages posted on the Bulletin Board using the scroll. & $7(4-7)$ & 90.9 \\
\hline I felt nervous to use the app. & $3(1-7)$ & 18.1 \\
\hline The application worked without any problems. & $6(2-7)$ & 72.7 \\
\hline
\end{tabular}

to walk or had a surgery on the knee or hip and, therefore, could not perform the recommended number of sets.

The ankle weights were a barrier to the adherence to the strength exercises because, apart from the effort required to lift the weights, most participants reported difficulties to wear and wrap them around their ankles. One man bought a new pair of weights that were easier to wear and close. Another woman mentioned "tying the weights is almost the hardest!"

Compliance with the intervention was excellent. Eleven of initially thirteen included elderly individuals completed the training. This is far more than the rate that could have been expected [35]. It should be noted, however, that the mean compliance rate for interventions in independent living elderly that was determined for several studies summarized in a systematic review [32] mainly focused on studies with far longer time periods. Our data should, therefore, be replicated in another study where the intervention is applied during several training sessions over several weeks. It can be expected that because of such a longer period less favorable compliance and retention rates will be achieved. Our findings warrant, however, such follow-up studies based on these first results.

Regarding motivation, we noticed that all participants felt motivated by making something grow with their effort and by receiving a simple reward (e.g., a new picture of the flower metaphor). We infer that positive reinforcement using the growing flower metaphor worked successfully, even if approximately half of the participants (55\%) would have preferred other metaphors. The selection of a metaphor (e.g., flower vs. car) is indeed very subjective and depends on the individual preferences of each participant.

About the low rate of writing activities, we hypothesize two possible reasons. Firstly, participants did not know each other, so they did not feel comfortable to write. Secondly, a minor part of them did not have good typing skills. For instance, one woman once wrote a non-understandable not correctly written message on the board. When she was asked about the sending messages option at the Bulletin Board or at the inBox, she said "I would write more, but for that I need to learn how to do it better." This 81 years old participant, however, never used the Internet or a computer before.

\subsection{Lessons learned}

We conclude that the participants enjoyed the training and felt motivated to follow the training plans using the iPadbased Active Lifestyle app. At the final meeting we received encouraging and motivating comments from the participants, e.g., their desire to continue using the app, their disappointment for not being able to continue performing the exercises without the app (the training plan finished, and they tried to 
continue performing after that). One woman, used to perform exercises with a book ("Fit after 40 "), commented, "the app is much better". To her the study was a much more motivating way to exercise. She and her husband took notes to continue the exercises even after the study. Another woman said that her doctor was impressed with the result of her cholesterol exam. The oldest man sustained a heart attack two years ago and stopped doing sports. According to him, "this was a great opportunity to reactivate" ". . . it was perfect. I noticed progress, especially on my balance". One person bought a tablet after the third meeting, and two expressed their intention to buy one soon and to install the app. However, because the test period was two weeks these findings are only preliminary. We cannot completely exclude the risk of the positive effect due to the news value of the app-based exercise program. The main focus was to evaluate the feasibility of the iPad-based intervention and the ability to recruit and retain elderly subjects. Home-based physical training with an iPad application seems to be feasible, as defined through recruitment, attrition and adherence to the exercise intervention. Our approach has shown to be a safe, low-cost and motivating way to activate and start with targeted physical exercise in older adults. To further clarify the influence of the iPad-based intervention on elderly subjects, for future studies we will consider a study design with a longer intervention period and a control group that will perform usual care exercises.

We learned that the motivational instruments worked. However, the possibility to chat did not motivate to use the Bulletin Board and the inBox features. Most participants were motivated being part of a training community, however, they were initially not willing to share their thoughts and feelings with strangers. We assume that if they learn how to use the app to make friends, receive feedback from people experiencing the same aging effects, and find a way to keep close and share their thoughts and life with old friends and family members, they might change their attitude. For that we need to build additional motivation and encouragement (i.e., dedicated mechanisms or features, in which the elderly have to reply questions, participate in collaborative activity or comment on specific topics). Another important aspect seems enabling communication and sharing of information between the elderly and their younger family members. During the intervention, we had only six comments on the Bulletin Board, of which three were addressed to our IT expert. We received sixteen private messages with suggestions and general comments about the exercises/app (e.g., "I am glad, my legs are not always so hard!" "The right leg is much stronger than the left leg! I feel that the training is necessary").

We also noticed that some of the participants felt very proud about using the tablet and shared this experience with their families. One woman showed the tablet and the app to her grandchildren, who were impressed: "grandma has an iPad, wow!" The oldest woman received hints from her daughter about how to perform the heel-to-walk exercise; she had difficulties to walk along a straight line. Finally, another participant invited her sister to perform the exercises together. These results reinforce our intention to enable family members to interact with the elderly via the app and to monitor their performance. We believe that social and emotional support can be a motivation instrument that should be accounted for in the revised version of our app.

\section{Limitations}

This study had some limitations. Because we wanted to assess the feasibility of the processes that are key to the success of a future main Phase III study [27] we decided to start with a short training period to avoid risks (e.g., late detection of bugs that can cause a higher participants withdrawal, high investment to provide the tablets and 3G SIM-card Internet connections). Due to that we did neither focus on recruitment strategies nor on outcomes of physical functioning. We were also not able to evaluate how the participants will respond to long-term use of the system. It can be hypothesized that, similar to observations made with devices such as the Wii Fit, persons will start to train enthusiastically in an initial phase and then abandon the use. However, we believe that an important difference between the Wii Fit training and our program is that ours contains dedicated physical training plans for elderly, designed by human movement scientists, who also monitor progress and support training participants, which turns the Active Lifestyle app a dedicated training software for elderly. In the next main Phase III study we should consider a longer training plan period (12-weeks) and also perform an assessment of the physical functioning status of participants, preferably with a pre-test/post-test randomized study design. From this pilot we can conclude, however, that sending letters to potentially qualifying elderly was not a good strategy to recruit participants.

This pilot study assessed the feasibility of our approach in a rather small sample, which might be considered another limitation. However, the purpose of Phase II trials, which may be randomized or not randomized [27], is to provide preliminary evidence on the clinical efficacy of an intervention. The processes of development, feasibility and piloting, evaluation and implementation are in nature iterative [27].

Another limitation of this study was the direct contact of our research and development team with the participants. It cannot be excluded that the participants were highly compliant to the training in an effort to please the researchers. However, at this initial test phase such contacts were necessary in order to understand the needs of the users and investigate what needs to be improved in the Active Lifestyle app.

\section{Conclusion}

We conclude that pilot studies with explicit feasibility objectives are important foundational steps in preparing for large trials. On-going formal review of the multifaceted issues inherent in the design and conduct of pilot studies can provide invaluable feasibility and scientific data for IT developers and rehabilitation specialists alike, and may be highly relevant for furthering the development of theory-driven, mobile-based rehabilitation.

This study shows that Active Lifestyle has potential to support physical exercise at home and it is worthwhile to further develop it into a more mature system. The results add to the 


\section{Summary points}

What was already known?

- A routine of strength and balance exercises contribute to prolong the independence of independently living elderly.

- ICT solutions have been largely adopted to track a routine of physical exercises among younger adults.

What this article added:

- The Active Lifestyle app successfully assists independently living elderly people to perform balance and strength exercises autonomously at home.

- The Active Lifestyle app motivated the participants to adhere a routine of physical exercises during the intervention period.

knowledge base on mobile-based applications for elderly, in that it shows that elderly users can learn to work with mobilebased systems. The Active Lifestyle app proves feasible to support and motivate independently living elderly adults to autonomously perform balance and strength exercises at home. The Active Lifestyle intervention in a main study is deemed feasible with some need for protocol modifications regarding recruitment strategies, motivational instruments and information on physical performance measures. In our further work we will more specifically examine the effectiveness of Active Lifestyle on measures of physical functioning, with longer training periods, and larger sets of participants. Additionally, we will investigate how the users of the Active Lifestyle app react to social and individual motivation factors, and compare their adherence and motivation against a control group.

\section{Author's contributions}

All authors participated during the conception and design of this study, as well as to the data analysis and interpretation, drafting and revising phases of this article. All authors contributed to drafting the manuscript, critically revising it for important intellectual content, and gave their approval of the final version to be submitted.

\section{Conflicts of interest statement}

None declared.

\section{Acknowledgements}

This work was carried out with the support of Maria MessmerCapaul of the Fachstelle für präventive Beratung Spitex-Zürich and Corinne Heck of the Informationsstelle fur Altersfragen. We would also like to thank all participants to the study for their great enthusiasm and support. This work is partially sponsored by the Trento Legge 6 project "Anchise".

\section{REFERENCES}

[1] W.W. Spirduso, D.L. Cronin, Exercise dose-response effects on quality of life and independent living in older adults, Med. Sci. Sports Exerc. 33 (6) (2001) 598-608 (PMID: 11427784).

[2] R.J. Johnson, F.D. Wolinsky, The structure of health status among older adults: disease, disability, functional limitation, and perceived health, J. Health Soc. Behav. 34 (2) (1993) 105-121 (PMID: 8277124).

[3] J.J. Malmberg, S.I. Miilunpalo, I.M. Vuori, et al., A health-related fitness and functional performance test battery for middle-aged and older adults: feasibility and health-related content validity, Arch. Phys. Med. Rehabil. 83 (5) (2002) 666-677 (PMID: 11994806).

[4] J. Malmberg, S. Miliunpalo, M. Pasanen, et al., Characteristics of leisure time physical activity associated with risk of decline in perceived health - a 10-year follow-up of middle-aged and elderly men and women, Prev. Med. 41 (1) (2005) 141-150 (PMID: 15917005).

[5] R. Beaglehole, S. Ebrahim, S. Reddy, et al., Prevention of chronic diseases: a call to action, Lancet 370 (9605) (2007) 2152-2157 (PMID: 18063026).

[6] D. Yach, C. Hawkes, C. Gould, et al., The global burden of chronic diseases: overcoming impediments to prevention and control, JAMA 291 (21) (2004) 2616-2622 (PMID: 15173153).

[7] P.T. Katzmarzyk, I. Janssen, The economic costs associated with physical inactivity and obesity in Canada: an update, Can. J. Appl. Physiol. 29 (1) (2004) 90-115, |(PMID: 15001807).

[8] W.J. Chodzko-Zajko, S.M. Fiatarone, C.T. Monson, et al., American College of Sports Medicine position stand. Exercise and physical activity for older adults, Med. Sci. Sports Exerc. 41 (7) (2009) 1510-1530 (PMID: 19516148).

[9] M. Stuart, S. Chard, F. Benvenuti, et al., Community exercise: a vital component to healthy aging, Healthc. Pap. 10 (1) (2009) 23-28 (PMID: 20057213).

[10] Anonymous (Ed.), Physical Activity Fundamental to Preventing Disease, U.S.D.o.H.H. Services, U.S. Department of Health \& Human Services, Washington, 2002.

[11] F. Landi, A.M. Abbatecola, M. Provinciali, et al., Moving against frailty: does physical activity matter? Biogerontology 11 (5) (2010) 537-545 (PMID: 20697813).

[12] M. Thibaud, F. Bloch, C. Tournoux-Facon, et al., Impact of physical activity and sedentary behaviour on fall risks in older people: a systematic review and meta-analysis of observational studies, Eur. Rev. Aging Phys. Act 8 (1.) (2011), DOI:10.1007/s11556-011-0081-1.

[13] J. Hilbe, E. Schulc, B. Linder, et al., Development and alarm threshold evaluation of a side rail integrated sensor technology for the prevention of falls, Int. J. Med. Inform. 79 (3) (2010) 173-180 (PMID: 20083427).

[14] Y. Mei, J. Marquard, C. Jacelon, et al., Designing and evaluating an electronic patient falls reporting system: Perspectives for the implementation of health information technology in long-term residential care facilities 2011. PMID: 21482183, in press.

[15] L. Ferrucci, S. Baldasseroni, S. Bandinelli, et al., Disease severity health-related quality of life across different chronic conditions, J. Am. Geriatr. Soc. 48 (11) (2000) 1490-1495 (PMID: 11083330).

[16] T. Obi, D. Ishmatova, N. Iwasaki, Promoting ICT innovations for the ageing population in Japan. Int. J. Med. Inform. 2012. PMID: 22727612, in press.

[17] H.J. Zunft, D. Friebe, B. Seppelt, et al., Perceived benefits and barriers to physical activity in a nationally representative 
sample in the European Union, Public Health Nutr. 2 (1A) (1999) 153-160 (PMID: 10933635).

[18] K.A. Schutzer, B.S. Graves, Barriers and motivations to exercise in older adults, Prev Med. 39 (5) (2004) 1056-1061 (PMID: 15475041).

[19] Anonymous. Physical Activity and Older Americans: Benefits and Strategies. Agency for Healthcare Research and Quality and the Centers for Disease Control. 2002. http://www.ahrq.gov/ppip/activity.htm

[20] J.K. Eckert, L.A. Morgan, N. Swamy, Preferences for receipt of care among community-dwelling adults, J. Aging Soc. Policy 16 (2) (2008) 49-65 (PMID: 15148044).

[21] C. Sherrington, J.C. Whitney, S.R. Lord, et al., Effective exercise for the prevention of falls: a systematic review and meta-analysis, J. Am. Geriatr. Soc. 56 (12) (2008) 2234-2243 (PMID: 19093923).

[22] J.A. Hess, M. Woollacott, Effect of high-intensity strength-training on functional measures of balance ability in balance-impaired older adults, J. Manipulative Physiol. Ther. 28 (8) (2005) 582-590 (PMID: 16226626).

[23] P. Heyn, B.C. Abreu, K.J. Ottenbacher, The effects of exercise training on elderly persons with cognitive impairment and dementia: a meta-analysis, Arch. Phys. Med. Rehabil. 85 (10) (2004) 1694-1704 (PMID: 15468033).

[24] O. Theou, L. Stathokostas, K.P. Roland, et al., The effectiveness of exercise interventions for the management of frailty: a systematic review, J. Aging Res. (2011) 569194 (PMID: 21584244).

[25] W.C. Mann, K.J. Ottenbacher, L. Fraas, et al., Effectiveness of assistive technology and environmental interventions in maintaining independence and reducing home care costs for the frail elderly. A randomized controlled trial, Arch. Fam. Med. 8 (3) (1999) 210-217 (PMID: 10333815).

[26] K. Laver, J. Ratcliffe, S. George, et al., Is the Nintendo Wii Fit really acceptable to older people? A discrete choice experiment, BMC Geriatr. 11 (2011) 64, DOI:10.1186/1471-2318-11-64.
[27] L. Thabane, J. Ma, R. Chu, et al., A tutorial on pilot studies: the what, why and how, BMC Med. Res. Methodol. 10 (1) (2010) 1-10 (PMID: 20053272).

[28] P. Craig, P. Dieppe, S. Macintyre, et al., Developing and evaluating complex interventions: the new Medical Research Council guidance, Brit. Med. J. 25 (337) (2008) 7676, DOI:10.1136/bmj.a1655.

[29] P. Silveira, F. Daniel, F. Casati, et al., Active Lifestyle: an application to help elderly stay physically and socially active. FoSIBLE Workshop at COOP2012, in press.

[30] LifeParticipation Project. 2012. http://www.lifeparticipation.org/active.html

[31] M.E. Cress, D. Buchner, T. Prohaska, et al., Best practices for physical activity programs and behavior counseling in older adults populations, J. Aging Phys. Act 13 (1) (2005) 61-74 (PMID: 15677836)

[32] W.J. Kraemer, N.A. Ratamess, Fundamentals of resistance training: progression and exercise prescription, Med. Sci. Sports Exerc. 36 (4) (2004) 674-688 (PMID: 15064596).

[33] Active Lifestyle app. 2012. http://www.youtube.com/watch?v=Akvs13UMvfc

[34] B.J. Fogg, Persuasive Technology: Using Computers to Change What We Think and Do, Morgan Kaufmann Series in Interactive Technologies, Boston, USA, 2008, ISBN:1-55860-643-2.

[35] S.R. Nyman, C.R. Victor, Older people's participation in and engagement with falls prevention interventions in community settings: an augment to the Cochrane systematic review, Age Ageing 41 (1) (2012) 16-23 (PMID: 21875865).

[36] M.J. Gibson, R.O. Andres, B. Isaacs, et al., The prevention of falls in later life - a report of the Kellogg International Work Group on the prevention of falls by the elderly, Dan. Med. Bull. 34 (1987) 1-24 (PMID: 3595217).

[37] F. Herzberg, B. Mausner, B.B. Snyderman, The Motivation to Work John Wiley, Transaction Publishers, New York, USA, 1959, ISBN:978-1560006343. 